\title{
E-ASSESSMENT OF RELATIONAL DATABASE SKILLS BY MEANS OF LEARNSQL
}

\author{
Carme Quer ${ }^{1}$, Alberto Abelló ${ }^{1}$, Xavier Burgués ${ }^{1}$, M. José Casany ${ }^{1}$, Carme \\ Martín $^{1}$, M. Elena Rodríguez ${ }^{2}$, Oscar Romero ${ }^{1}$, Toni Urpí ${ }^{1}$ \\ ${ }^{1}$ Universitat Politècnica de Catalunya (SPAIN) \\ ${ }^{2}$ Universitat Oberta de Catalunya (SPAIN)
}

\begin{abstract}
LearnSQL is a software system that allows the automatic and efficient e-learning and e-assessment of relational database skills. It has been used at the Barcelona School of Informatics for 18 semesters with an average of 200 students per semester. This paper shows the functionalities of LearnSQL subsystems by means of specific and understandable examples.
\end{abstract}

Keywords: Computing Engineering Education, Interactive Learning Environments, Improving Classroom Teaching, Blended Learning

\section{INTRODUCTION}

The increase of several forms of e-learning in the last few years (i.e., Massive Open Online Courses) has fostered the emergence of e-assessment systems to support the automatic evaluation of students' responses to proposed exercises. At the Barcelona School of Informatics (FIB) of Universitat Politècnica de Catalunya-BarcelonaTech the e-assessment is especially relevant due to the profile of the students and the nature of the studies.

In database related courses, students require analytical, creative, and constructive skills that cannot be assessed via multiple-choice tests or equivalent forms of basic assessment techniques. From a technological point of view, this requires more complex e-assessment systems. LearnSQL (Learning Environment for Automatic Rating of Notions of SQL) [1] is a software system that allows the automatic and efficient e-learning and e-assessment of relational database skills. It has been used at FIB for 18 semesters with an average of 200 students per semester.

LearnSQL encourages learning by providing students with exercises to practice and learn the studied topics. Teachers prepare tests that are to be solved during face-to-face laboratory sessions and other tests that are to be solved at home. At laboratory sessions, the students get used to the learning environment. The students, organized in collaborative teams, solve exercises thanks to the immediate feedback provided after submitting their responses and, if necessary, with the teachers' advice. Both at laboratory sessions and at home, the students can make as many trials as necessary to solve the exercises.

From the assessment perspective, LearnSQL supports the e-assessment of the students' learned skills by automatically assessing and grading the students' responses. The teachers prepare exam tests using LearnSQL. When the students come up with a possible response, they send it to LearnSQL to be assessed. Additionally, the teachers can limit the number of submissions during the exams, and a penalty can be applied in case of multiple trials. Thus, the resulting grade is an indicator of the students' learned skills.

The architecture of LearnSQL is composed of four subsystems (Fig. 1): the Authoring Tool is used by teachers to create and manage exercises; the Remote Test Module is used by students to solve exercises; the Team Creator Module is used by teachers and students to create teams of students; the Scorer implemented in the form of a web service for assessing exercise responses. The architecture of LearnSQL is compliant with the IMS QTI specification [2], which enables the system automatization, interactivity, informativeness, scalability and extensibility.

This paper shows the functionalities of LearnSQL subsystems by means of specific and understandable examples.

Quer, C., Abelló, A., Burgués, X., Casany, M.J., Martín, C., Rodríguez, M.E., Romero, O., Urpí, T. E-assessment of relational database skills by means of LearnSQL. A: International Conference on Education and New Learning Technologies. "EDULEARN17 proceedings: 9th International Conference on Education and New Learning Technologies: Barcelona, Spain, 3-5 July, 2017". Barcelona: International Association of Technology, Education and Development (IATED), 2017, p. 9443-9448. DOI 10.21125/edulearn.2017.0779 


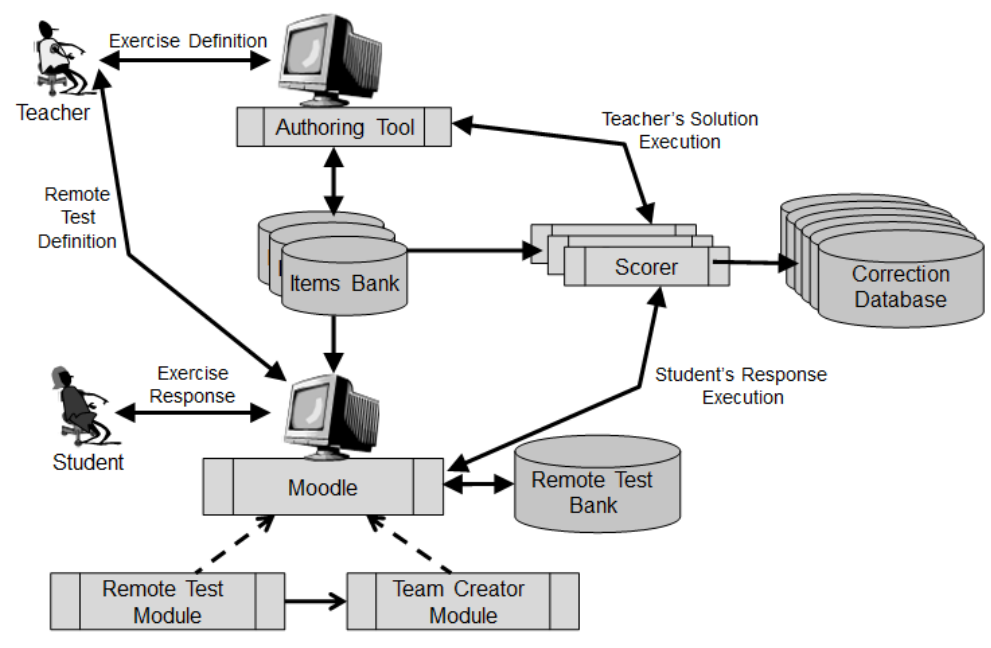

Figure 1. LearnSQL subsystems

\section{AUTHORING TOOL}

The Authoring Tool (AT) is a subsystem used by the teachers for creating and managing exercises. Each exercise created is related to a certain database schema, corresponds to a certain predefined type of exercise, and is used to practice one or more thematic.

One teacher that wants to create a new exercise has to firstly introduce the general attributes of the exercise. The concepts in the definition of an exercise are the same independently of the type of exercise (see a conceptual diagram in Fig. 2). The most relevant are the following: the exercise Statement in natural language, the Initialization and Cleaning sentences of the database (Fig. 3), a File provided to the student that contains different material depending on the type of exercise, and the Scorer (SCR) that will be used to assess and correct the student responses to the exercise.

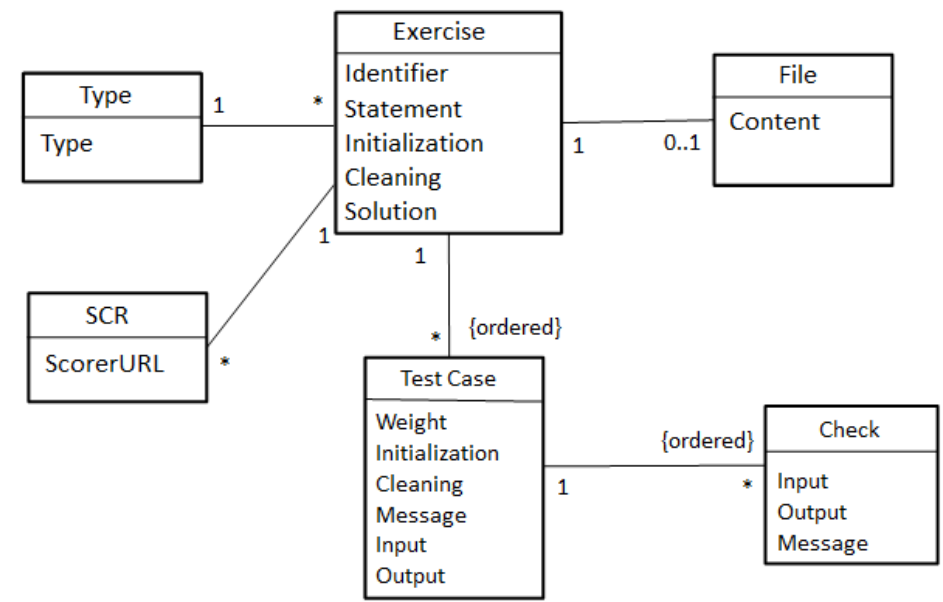

Figure 2. Concepts in the LearnSQL

The assessment of exercises in LearnSQL is based in the definition of multiple Test Cases. Each test case corresponds to a different state of the database that is relevant for the exercise and has a Weight that is assigned by the teacher based on the total weight of the exercise. In the definition of each test case, it is necessary to define the Initialization and Cleaning sentences. In some types of exercises, the teacher needs to define one or more Checks for each test case in order to query the state of the database after the changes produced by the execution of the student's response. The teacher is responsible for the introduction of the Message associated to each test case that will be the feedback provided to the student if the test case or check is not passed successfully. In some types of exercises, the teacher also needs to define an Input for each test case. 
To finish the definition of one exercise the teacher introduces a correct Solution (i.e., the teachers' solution) for the exercise. The solution is executed by the SCR associated to the exercise (the SCR configuration states the DBMS and database where it is executed) to generate correct Outputs for each Test Case and each Check.

During the conference we will show how to define an exercise.

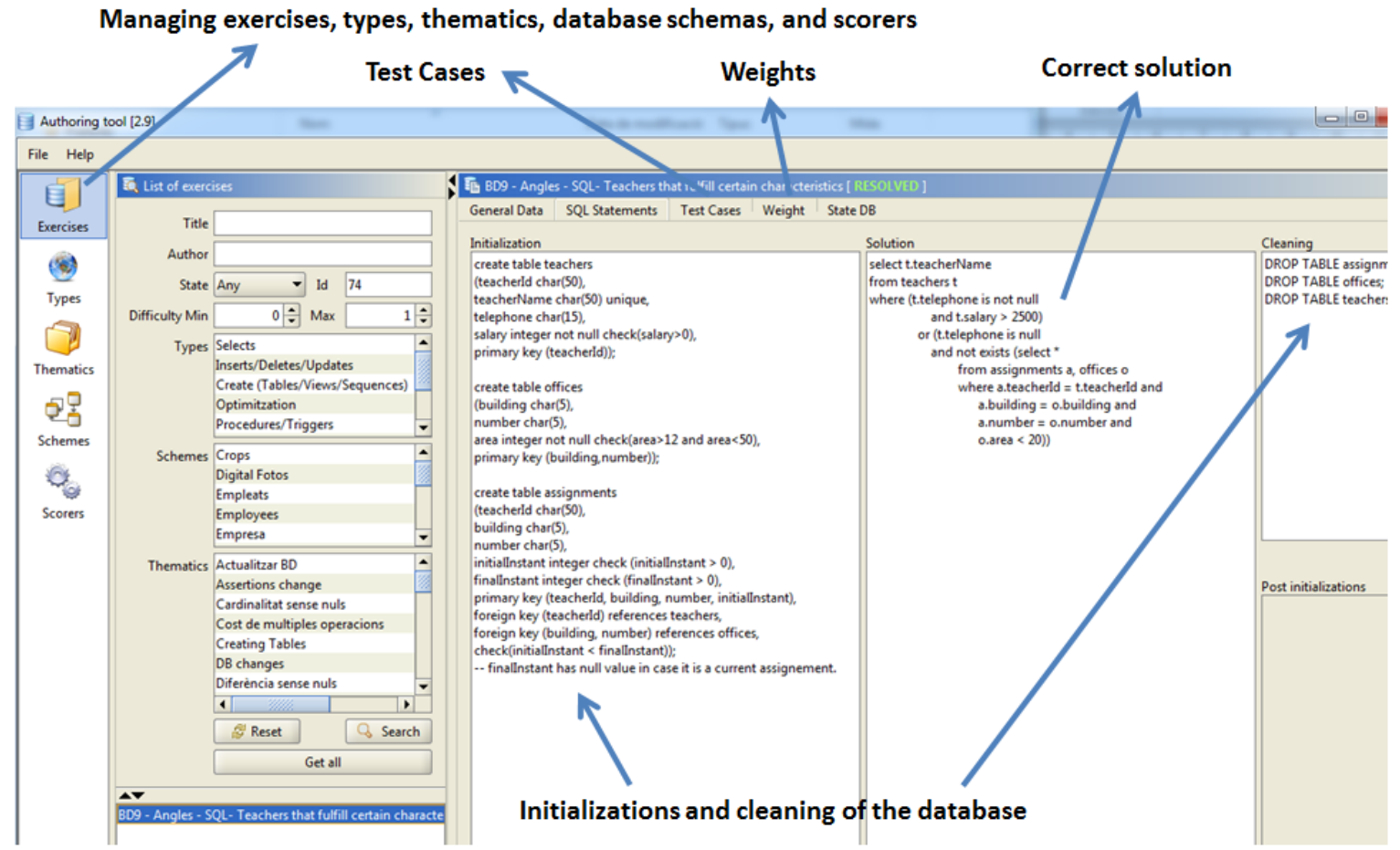

Figure 3. Authoring Tool

\section{REMOTE TEST MODULE}

The Remote Test Module (RTM) is used by students to solve exercises. This module also provides functionalities to teachers, such as grouping exercises in tests, monitoring students' progress, publishing grades, and giving feedback to the students, etc. The RTM is implemented as an activity module that extends Moodle. Moodle provides the LearnSQL system with the Learning Management System (LMS) infrastructure to manage courses, groups, students, teachers, etc.

After one or more exercises are defined, by means of the AT, the teacher may group the exercises in remote tests. During the definition of a test the teacher states the position of each exercise in the test and its weight with regard the grade of the test.

Fig. 4 shows the presentation of an exercise to the students. The students see: the weight of the exercise in the test (in the example, $30 \%$ of the total grade of the test); the statement that defines what the student is required to do (write an SQL sentence); the result of executing the teacher's solution for the public test case provided in the attached file (the teacher Toni); a text area to enter the student's response; the "Submit" button to correct the response and obtain feedback; the link to a pop-up window with the student's response that has been graded; and the current grade, the number of the next submission, and the total number of submissions allowed (grade for the submission is 7.2, if the student retries the exercise next submission will be the third one, 10 submissions allowed). Finally, the feedback of the correction process of the last response that has been submitted ("TC5: you have probably missed a join", therefore just test case TC5 failed). 


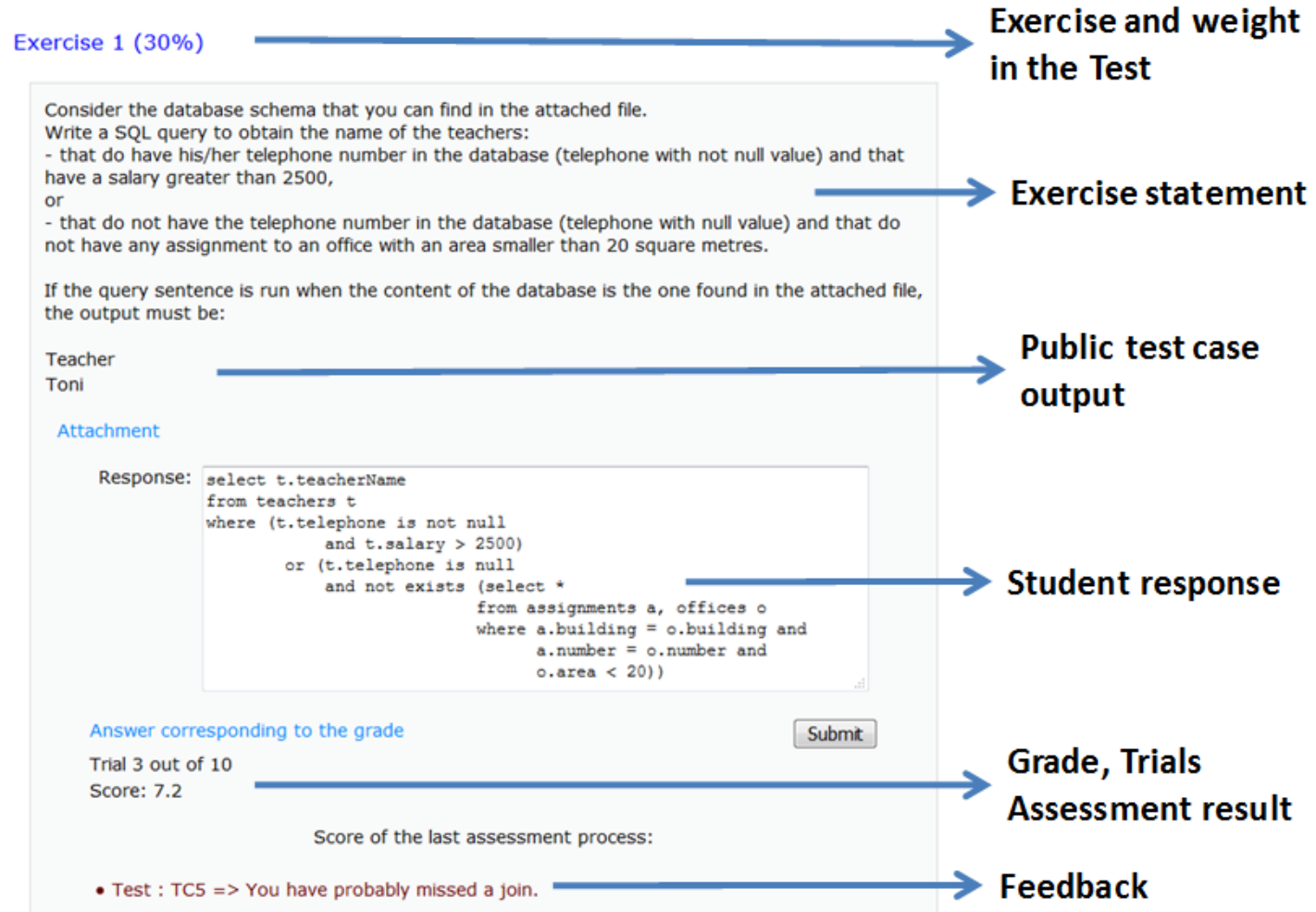

Figure 4. SQL Query exercise example

During the conference we will show how a student uses LearnSQL during laboratory sessions, during home work or exams.

\section{SCORER}

The Scorer (SCR) is a web service that corrects exercise responses. This scorer is required by the AT when defining of new exercises. It is also required by the RTM during the correction stage of the students' responses. The SCR configuration allows setting the specific DBMS that is used in the course (the DBMS used to date are PostgreSQL, Oracle, and SQL Server), in which the exercise responses will be assessed. The SCR is currently implemented as a SOAP web service. There exist five algorithms that the scorer selects depending on the type of exercise assessed (Table 1).

Table 1. Types of exercises

\begin{tabular}{|ll|}
\hline LearnSQL Algorithms & Types of exercises \\
\hline Algorithm 1 & SQL Queries \\
& SQL Ins/Del/Upd \\
& DDL \\
& Logical Design \\
& Normalization \\
& Relational Algebra \\
& Multidimensional Operations \\
& Optimization using Materialized \\
& views $\quad$ SQL/PSM \\
\hline Algorithm 2 & Triggers \\
\hline Algorithm 3 & Programming with SQL (JDBC) \\
\hline Algorithm 4 & Optimization using Indexes \\
& Optimization of Workloads \\
\hline Algorithm 5 & Cost Estimation \\
\hline
\end{tabular}

\section{TEAM CREATOR MODULE}

The Team Creator Module (TCM) is used by teachers and students to create teams of students. LearnSQL provides the possibility of solving tests collaboratively and to interact with the system as a 
team. When a test is created the teacher may decide whether it must be solved individually or by teams. If the exercises are to be solved by teams, the responses and grades of the exercises are associated to all the team members. The teams may be composed of two or more students. As in the case of RTM, the TCM is implemented as another module extending Moodle.

When a teacher decides that an activity will be solved by teams, there are three options for creating them (Fig. 5). One is to automatically generate the teams. The automatic generator builds the teams by selecting randomly the students of the course. The teacher may define some additional criteria that are taken into account during the generation. The second option for creating the teams is that the teacher decides their composition. And, finally, the last option is to allow the students to decide their teammates.

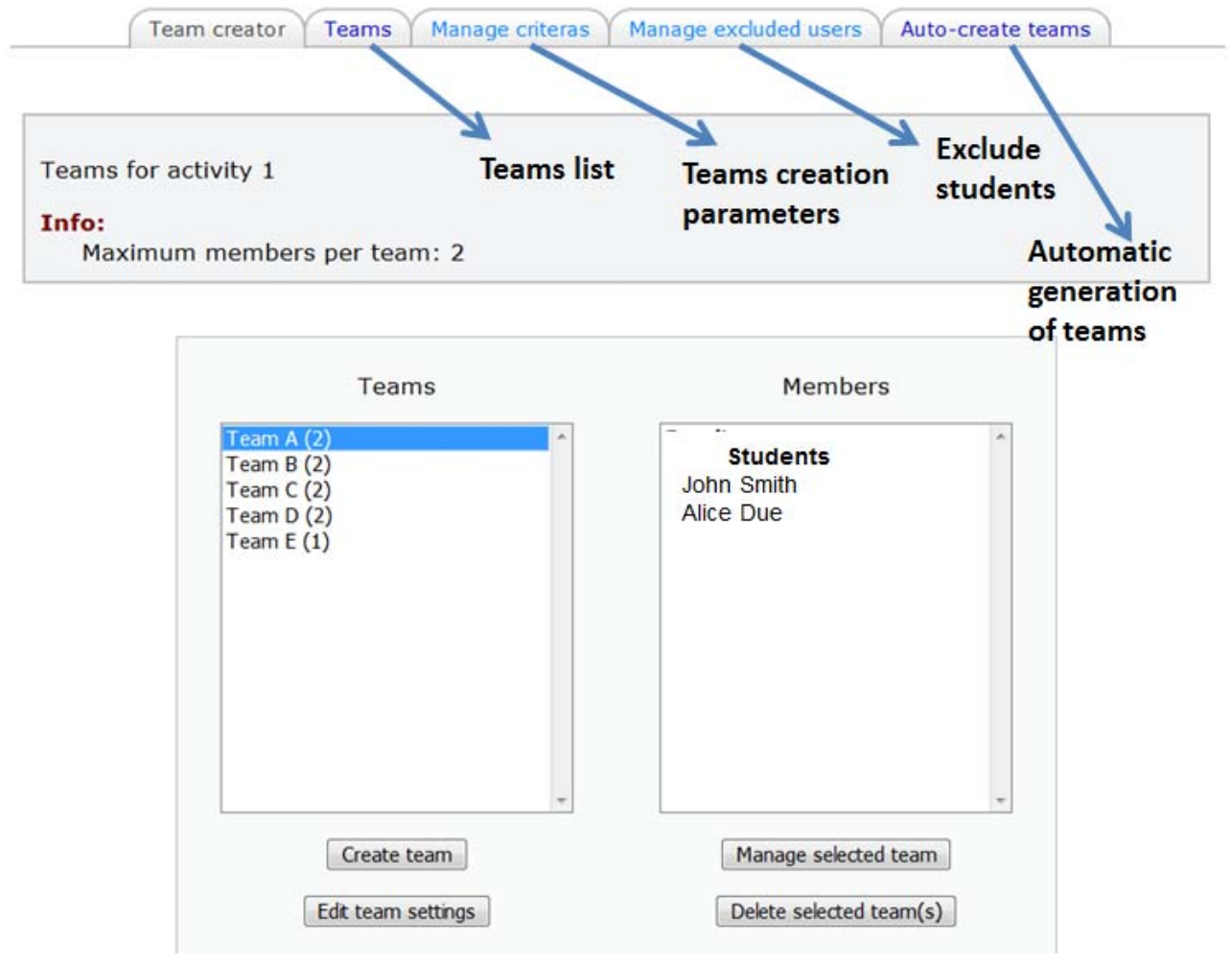

Figure 5. Teams creation

During the conference we will show how teams may be generated and used from the RMT.

\section{FINAL REMARKS}

In [1] we detail the main advantages of LearnSQL from the teachers' and students' point of view. We also include the results of the qualitative and quantitative analyses of the use of LearnSQL in FIB database courses. The authors finally provide a set of lessons learned based on their experience that may be helpful to teachers considering introducing e-assessment in their courses.

\section{ACKNOWLEDGEMENTS}

We are grateful to FIB for its contribution for the presentation of this work in EDULEARN 2017.

\section{REFERENCES}

[1] Alberto Abelló, Xavier Burgués, M. José Casany, Carme Martín, Carme Quer, M. Elena Rodríguez, Oscar Romero, Toni Urpí: A Software Tool for E-Assessment of Relational Database Skills. International Journal of Engineering Education. 32, 3(A), 2016.

[2] IMS Global Learning Consortium, IMS Question \& Test Interoperability Specification. https://www.imsglobal.org/. Accessed March 2017. 